\title{
An Acceptor-m-Donor Structured Organic Chromophore for NIR Triggered Thermal Ablation of Tumor via DNA Damage-Mediated Apoptosis
}

\author{
Di Zhang' \\ Jinghong Yang ${ }^{2}$ \\ Chuang Liu' \\ Sheng $\mathrm{Ye}^{\prime}$ \\ Qianbing Zhang ${ }^{2}$ \\ Ruiyuan Liu (D)' \\ 'Guangdong Provincial Key Laboratory of \\ Medical Image Processing, School of \\ Biomedical Engineering, Southern Medical \\ University, Guangzhou, Guangdong, \\ 5I05I5, People's Republic of China; \\ ${ }^{2}$ Cancer Research Institute, School of \\ Basic Medical Sciences, Southern Medical \\ University, Guangzhou, Guangdong, \\ 5I05I5, People's Republic of China
}

Correspondence: Ruiyuan Liu Guangdong Provincial Key Laboratory of Medical Image Processing, School of Biomedical Engineering, Southern Medical University, Guangzhou, Guangdong, 510515, People's Republic of China Tel/Fax +86-20-6I647496

Email ruiyliu@smu.edu.cn

Qianbing Zhang

Cancer Research Institute, School of Basic Medical Sciences, Southern Medical University, Guangzhou, Guangdong,

5 10515, People's Republic of China

Tel/Fax +86-20-61648378

Email carl@smu.edu.cn

\begin{abstract}
Introduction: It will be challenging to develop high-performance organic chromophores for light-triggered thermal ablation of the tumor. Besides, the mechanisms of organic chromophores for tumor therapy remain unclear. Herein, an acceptor- $\pi$-donor (A- $\pi$-D) structured organic chromophore based on 2-dicyanomethylenethiazole named PTM was developed for photothermal therapy (PTT) of tumors.

Methods and Results: Biocompatible PTM nanoparticles (PTM NPs) were fabricated by enclosing PTM with Pluronic F-127. The results of optical and photothermal properties of PTM NPs showed robust near-infrared (NIR) absorption, excellent photostability and high photothermal conversion efficiency $(56.9 \%)$. The results of flow cytometry, fluorescence microscopy, apoptosis, CCK-8 assays and animal experiments showed that PTM NPs had a good killing effect on tumors under NIR laser irradiation. Furthermore, mechanistic studies, RNA-seq and biological analysis revealed that PTM NPs can cause tumor cell death via DNA damage-mediated apoptosis.
\end{abstract}

Conclusion: Light-induced thermal ablation effects of PTM NPs in vitro and vivo were surveyed. Collectively, our studies provided a new approach to developing a safe and effective photothermal agent for cancer treatment.

Keywords: NIR absorbing chromophore, 2-dicyanomethylenethiazole, photothermal therapy, DNA damage induced apoptosis, RNA-seq, biological analysis

\section{Introduction}

Thermal ablation of tumors is an application of hyperthermia, which will result in irreversible cell injury, the tumor apoptosis and coagulative necrosis ultimately. ${ }^{1,2}$ At present, several thermal ablation methods have been developed, including radiofrequency ablation (RFA), ${ }^{3,4}$ microwave ablation (MWA) ${ }^{5,6}$ and high-intensity focused ultrasound (HIFU). ${ }^{7,8}$ However, expensive instruments were used in these methods. Compared with methods mentioned above, non-invasive photothermal therapy (PTT) be more economical, more effective, more precise targeting at the tumor, and minimize the damage to surrounding healthy tissues. PTT can kill cancer cells and bacterial biofilm via heat energy generated by photothermal agents under NIR laser irradiation. ${ }^{9-15}$ In recent years, various inorganic nanomaterials have been actively employed for photothermal therapy of tumors, including gold nanoparticles, ${ }^{16}$ carbon nanomaterials, ${ }^{17}$ palladium nanosheets, ${ }^{18}$ and transitional 


\section{Graphical Abstract}

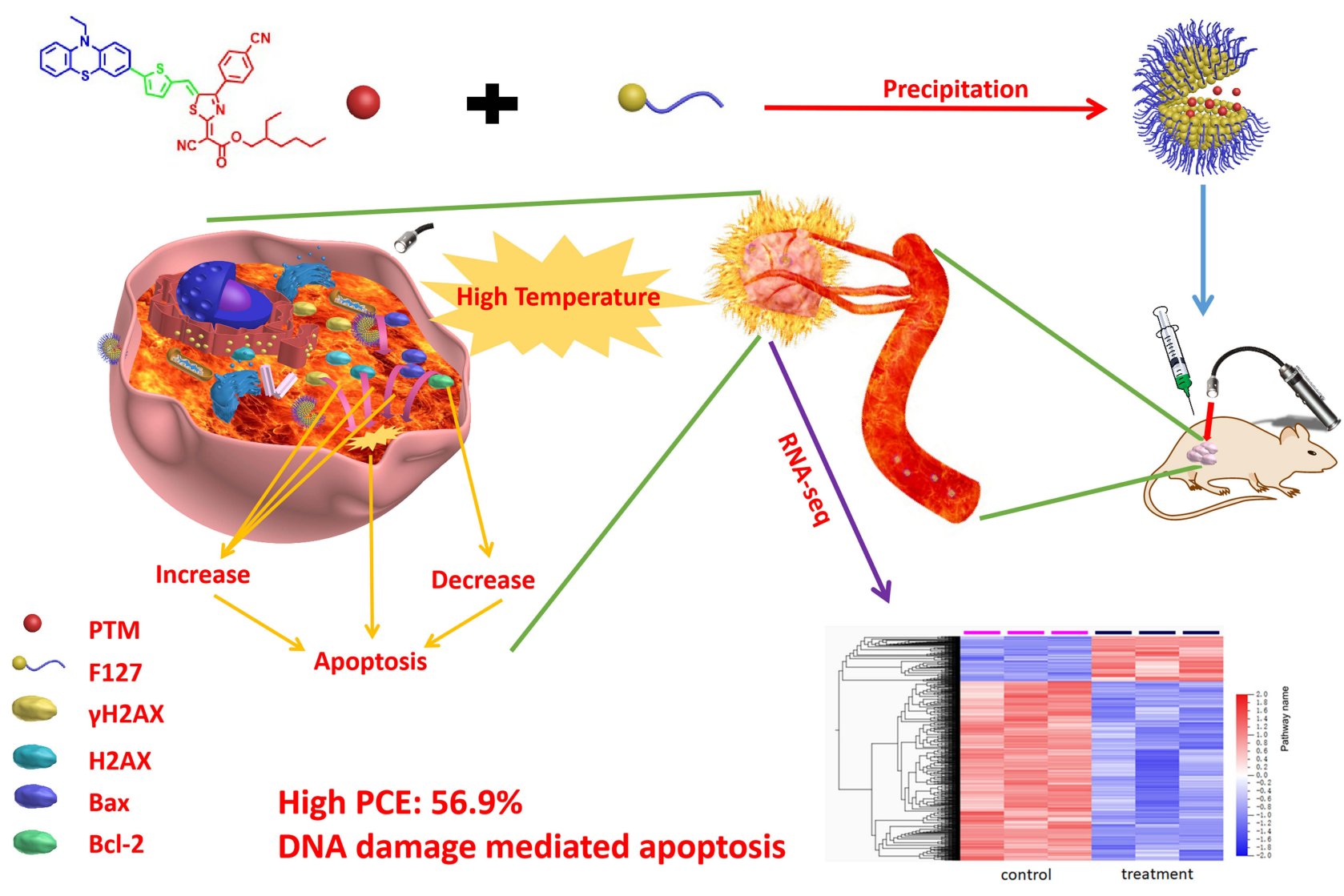

metal dichalcogenide. ${ }^{19,20}$ Although superior efficiency in ablation tumors has been achieved by using inorganic photothermal agents, the potential long-term toxicity and poor biodegradation remain the major challenges. On the other hand, the NIR-absorbing (750 1700 nm) organic photothermal agents have been explored owing to their great biocompatibility and can be metabolied rapidly in biological tissue, including NIR absorbing conjugated polymers, $^{21}$ cyanines $^{22}$ and porphyrins. ${ }^{23}$ However, the poor photostability, severe photodegradation, and the low efficiency of photothermal conversion hamper their further applications. Dibenzamide dyes exhibit excellent molar absorption and subtly tunable optical properties, but their biological applications are mainly focused on fluorescence imaging, ${ }^{24}$ their maximum absorption and emission in the visible range are limited. Terrylenediimide-based nanomedicines have high photothermal conversion efficiency, but the preparation process with certain inaccuracies is complex and unstable..$^{25,26}$ Hence, it is crucial to develop a new class of NIR absorbing organic chromophores for thermal ablation in tumor.
A- $\pi$-D structured organic chromophores have been demonstrated to be an excellent option for photothermal therapy due to their excellent photostability and strong molar absorption coefficient. ${ }^{27-30}$ Besides, chromophores with $A-\pi-D$ structure have rigid planar structures and strong $\pi-\pi$ interaction, which enhance the non-radiative decay and improve photothermal efficiency. ${ }^{31}$ To date, efforts have been made to improve the PTT efficacy of A- $\pi$-D structured organic chromophores in many researchs, the strategies proposed including increasing acceptor strength, extending conjugation length to reduce band gap, and red-shift the absorption maxima. ${ }^{32}$ To this effect, numbers of electron-deficient groups have been exploited, including diketopyrrolopyrrole, ${ }^{33,34}$ thiadiazolobenzotriazole, ${ }^{35,36}$ benzo[1,2-c:4,5c']bis([1,2,5] thiadiazole), ${ }^{37,38}$ and indan. ${ }^{39,40}$ Among them, 2-dicyanomethylenethiazole, an electron-withdrawing core, can be combined with the electron-donating group to build an A- $\pi$-D organic chromophore for broadening the absorption spectrum, reducing the optical gap, and decreasing the HOMO level. $^{41-45}$ However, 2-dicyanomethylenethiazole 
molecules exhibit some limitations, such as weak absorbance in the near-infrared (NIR) region and low photothermal conversion efficiency. Therefore, it is highly desirable to explore new 2-dicyanomethylenethiazole chromophores with high PTT performance.

As reported, the hyperthermia treatment can inhibit cancer cells tumorigenesis via the necroptosis pathway or DNA damage-mediation tumor apoptosis pathway. ${ }^{46-51}$ However, the underlying mechanisms are still elusive. The comprehensive understanding of photothermal agents might provide new options for efficient cancer therapy. As shown in Graphical Abstract, in this contribution, we developed an A- $\pi$-D structured organic chromophore (PTM) based on 2-dicyanomethylenethiazole for lightinduced tumor thermal ablation. PTM was directly encapsulated into an amphiphilic copolymer Pluronic F-127 through the nanoprecipitation method to obtain nanoparticles, PTM NPs. These agents shown robust photostability, good biocompatibility, and high PCE (56.9\%). Moreover, PTM NPs exhibited cancer cells killing capacity via DNA damage mediated apoptosis induced by heat generation in cells (Scheme 1). Both in vitro and in vivo experiments demonstrated that PTM NPs had effective anti-tumor potential. Our comprehensive studies of the mechanisms and underlying PTM NPs performance will improve the understanding of photothermal therapy and augment the future application of photothermal agents.

\section{Materials and Methods}

\section{Chemicals and Materials}

All reagents and solvents were commercially provided. CCK-8 kit was provided from Dojindo Laboratories (Kumamoto, Japan). Calcein AM/PI Detection Kit and Hematoxylin and Eosin Staining Kit were purchased from NanJingKeyGen Biotech Co., Ltd. (China). 5-(10Ethyl-phenothiazin-3-yl)thiophene-2-carbaldehyde was prepared according to reference. ${ }^{52}$

\section{Design and Synthesis of PTM}

5-(10-Ethyl-phenothiazin-3-yl)thiophene-2-carbaldehyde (3.36 g, $10 \mathrm{mmol})$ and 2-dicyanomethylenethiazole 1 (3.82 $\mathrm{g}, 10 \mathrm{mmol}$ ) and was added into $30 \mathrm{~mL}$ acetic anhydride, and stirred at $160{ }^{\circ} \mathrm{C}$ for $12 \mathrm{~h}$ under $\mathrm{N}_{2}$ protection. The mixture was drop added into saturated $\mathrm{Na}_{2} \mathrm{CO}_{3}$ aq. The dark blue solid was filtrated and purified by column chromatography using a mixture of DCM/MeOH (19: 1). Yield: 4.8 g (67\%). ${ }^{1} \mathrm{H}$ NMR (400 MHz, $\left.\mathrm{CDCl}_{3}\right), \delta(\mathrm{ppm}): 7.86(\mathrm{~s}, 1 \mathrm{H})$,
$7.67 \sim 7.69(\mathrm{~d}, 2 \mathrm{H}), 7.57(\mathrm{~s}, 1 \mathrm{H}), 7.45 \sim 7.47(\mathrm{~d}, 2 \mathrm{H}), 7.40 \sim 7.42$ (d, 2H), 7.32 7.36(d, 2H), 7.28(s, 1H), 7.18 7.24(t, 2H), 6.94 7.05(m, 2H), 6.81(s, 1H), 3.81 3.84(d, 2H), 3.16 3.19 (t, 2H), 1.59(s, 1H), 1.31 1.49(m, 8H), 0.91 0.95(m, 9H). ${ }^{13} \mathrm{C}$ NMR (100 MHz, $\left.\mathrm{CDCl}_{3}\right), \delta(\mathrm{ppm}): 172.16,166.78$, $163.41,148.70,147.68,146.71,143.83,141.58,141.16$, $137.38,136.40,134.27,133.11,132.03,131.28,130.60$, $129.31,128.16,127.85,126.54,124.43,121.11,120.18$, $119.30,115.98,115.12,111.26,95.94,64.48,49.56,44.41$, $31.76,29.14,26.55,26.04,15.83,15.04,14.33$. IR $\left(\mathrm{v}^{-1}\right.$ $\mathrm{KBr}): \mathrm{cm}^{-1} 3453,2986,2845,2618,1618,1523,1483$, $1366,1252,1034,863$.

\section{PTM NPs Preparation}

PTM NPs were constructed by the coprecipitation method. Pluronic F-127 (100 mg) and PTM (10 mg) were mixed in THF $(1 \mathrm{~mL})$. THF solution was drop added into DI water $(10 \mathrm{~mL})$ under ultrasonic condition. The mixture was bubbling $\mathrm{N}_{2}$ to remove THF completely. PTM was obtained after dialysis to remove the free F-127.

\section{Animals}

All the animal experiments were conducted in accordance with the institutional guidelines for the care and use of laboratory animals at Southern Medical University, Guangzhou, China, and Regulations for the Administration of Affairs Concerning Experimental Animals (1991.7, revised 2017). All the animal experiments have been approved by Southern Medical University.

\section{Statistical Analysis}

All data were expressed as means \pm standard deviation (SD) or means \pm standard errors. All figures shown in this article were obtained from at least three independent experiments. Analysis of variance was employed for multiple group comparisons, and results of $p<0.05$ were considered statistically significant.

\section{Result and Discussion Synthesis, Optical and Photothermal Property of PTM NPs}

NIR absorption for A- $\pi$-D structured organic chromophore (PTM) was built by using 2-dicyanomethylenethiazole as a strong acceptor, allowing for greater electron delocalization and thus lowering the band gap, phenothiazine as a donor, and planar thiophene ring as a $\pi$-conjugation unit, which 
provided effective conjugation and large extinction coefficient. The synthetic routine of PTM is shown in Scheme S1 and $\underline{\mathrm{S}}$. The synthesis process, NMR spectrum, and IR spectrum are shown in Figure S1-S5 (Supporting information).

To further enhance the biocompatibility, hydrophobic PTM and Pluronic F-127 were dissolved in THF and allowed to self-assemble into PTM NPs. First of all, the absorption property of PTM NPs was evaluated, as shown in Figure 1A, PTM NPs $(200 \mu \mathrm{g} / \mathrm{mL})$ have a broad UV-vis absorption arrange from $550 \mathrm{~nm}$ to $1000 \mathrm{~nm}$ with the maximum absorption located at $\sim 680 \mathrm{~nm}$, which permits deep tissue penetration and exhibits the potential to accomplish efficient photothermal therapy for deep tumor. Furthermore, the dynamic light scattering (DLS) experiment showed that PTM NPs exhibited hydrodynamic diameters around $110 \mathrm{~nm}$ (Figure 1B). Interestingly, the size of PTM NPs in PBS keeps stable within
28 days, which confirms the robust stability (Figure S6). In addition, TEM and SEM were measured to confirm the morphology and size of PTM NPs (Figure S7-S8).

The light-induced thermal activity of PTM NPs was also explored. The relation between laser power and temperature variation of PTM NPs in PBS was surveyed (Figure 1C). The temperature of PTM NPs solutions increased quickly with improving the laser power, confirming that the temperature elevation is proportional to the laser power density. As depicted in Figure 1D, when the concentration of PTM NPs reached $160 \mu \mathrm{g} / \mathrm{mL}$, the solution temperature rose rapidly to $72.4^{\circ} \mathrm{C}$ under NIR laser irradiation $\left(808 \mathrm{~nm}, 1.5 \mathrm{~W} / \mathrm{cm}^{2}, 10 \mathrm{~min}\right)$. It is worth noting that PTM NPs also reached effective hyperthermia $\left(45^{\circ} \mathrm{C}\right)$ with the concentration of $40 \mu \mathrm{g} /$ $\mathrm{mL}$ under the same conditions, demonstrated that PTM
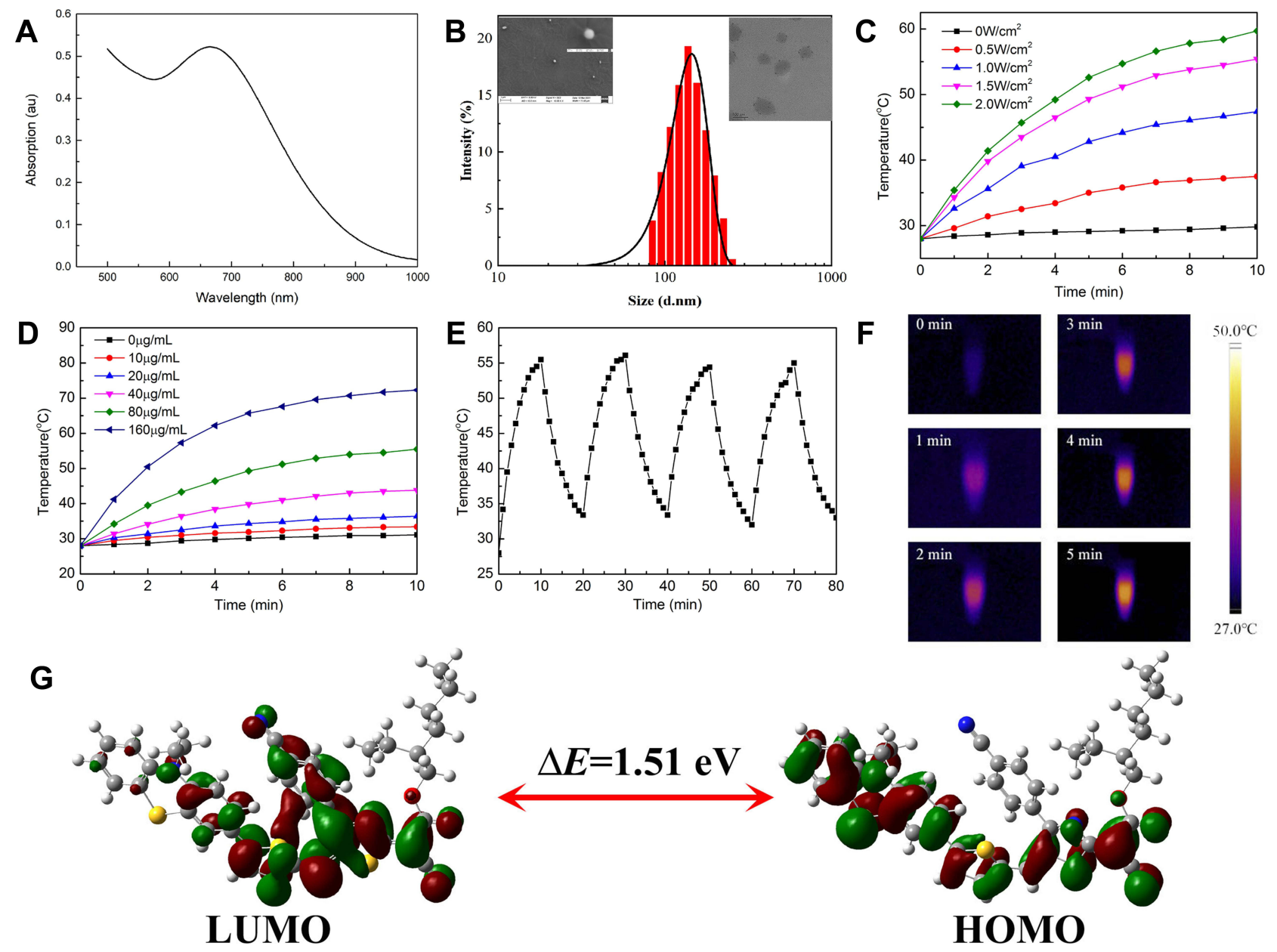

Figure I (A) The absorption spectrum of PTM NPs $(200 \mu \mathrm{g} / \mathrm{mL})$ in Water. (B) DLS of PTM NPs $(10 \mu g / \mathrm{mL})$ insert SEM and TEM of PTM NPs. (C) Photothermal heating curves of PTM NPs $(80 \mu \mathrm{g} / \mathrm{mL})$ irradiated for $10 \mathrm{~min}$ using a $808 \mathrm{~nm}$ laser at varied power densities $\left(0,0.5,1.0,1.5\right.$, and $\left.2.0 \mathrm{~W} / \mathrm{cm}^{2}\right)$. (D) The temperature evolution of PTM NPs with different concentrations under $808 \mathrm{~nm}$ laser irradiation ( $\left.1.5 \mathrm{~W} / \mathrm{cm}^{2}, 10 \mathrm{~min}\right)$. (E) Temperature elevation of PTM NPs (I $\left.20 \mu \mathrm{g} / \mathrm{mL}\right)$ under five on $/$ off cycles (I.0 W/ $\left.\mathrm{cm}^{2}\right)$. (F) Corresponding near infrared photographs of PTM NPs $(80 \mu \mathrm{g} / \mathrm{mL})$ under NIR laser irradiation $\left(808 \mathrm{~nm}, 1.5 \mathrm{~W} / \mathrm{cm}^{2}, 5 \mathrm{~min}\right)$. (G) $\mathrm{HOMO}$ and LUMO calculated by DFT at the B3LYP/6-3IG (d,p) basis set. 
NPs displayed excellent photothermal properties even under a low concentrations.

The photothermal conversion efficiency of PTM NPs was evaluated to be $\sim 56.9 \%$ according to the reported method (Figure S9), ${ }^{53}$ which is significantly superior to most previously reported photothermal reagents, including porphyrin $(62.5 \%))^{54}$ phthalocyanine-based nanodots $(45.7 \%),{ }^{55}$ cyanine dyes $(26.6 \%),{ }^{56}$ polypyrrole NPs $(45 \%),{ }^{57}$ dopamine-melanin NPs $(40 \%),{ }^{58} \mathrm{Au}$ nanorods $(21.0 \%),{ }^{59} \mathrm{MoS}_{2}$ nanosheets $(24.4 \%) .{ }^{60}$ All these results confirmed that PTM NPs can act as a promising candidate for photothermal therapy.

The photostability of PTM NPs was also investigated. The temperature changes of PTM NPS solution $(120 \mu \mathrm{g} /$ $\mathrm{mL}$ ) were continuously monitored under NIR laser irradiation $\left(808 \mathrm{~nm}, 1.0 \mathrm{~W} / \mathrm{cm}^{2}, 10 \mathrm{~min}\right)$ and then cooling to r.t. naturally for five heating/cooling cycles. As shown in Figure 1E, the photothermal capacity changes slightly. The photobleaching stability of PTM NPs was further determined by continuous irradiation of PTM NPs in PBS $(120 \mu \mathrm{g} / \mathrm{mL})$ upon NIR laser decreased slightly, and no obvious color change was observed (Figure S10). These results confirmed that PTM NPs have excellent stability against photobleaching. The temperature of PTM NPs in aqueous dispersions was also investigated upon NIR laser irradiation (808 $\left.\mathrm{nm}, 1.5 \mathrm{~W} / \mathrm{cm}^{2}, 5 \mathrm{~min}\right)$. Infrared thermal images after irradiation were also present in Figure 1F.
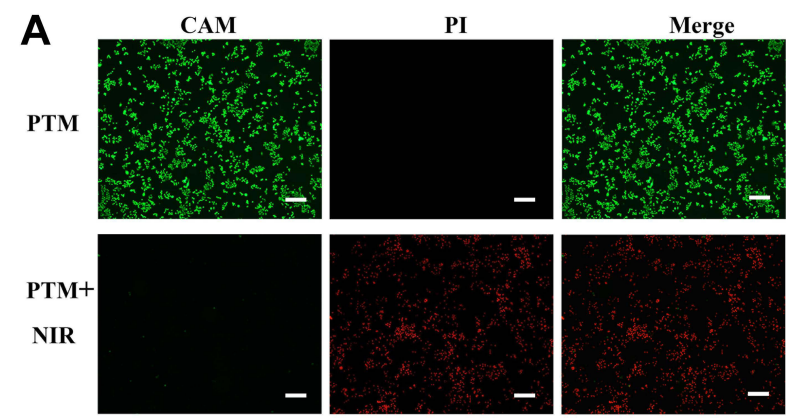

B

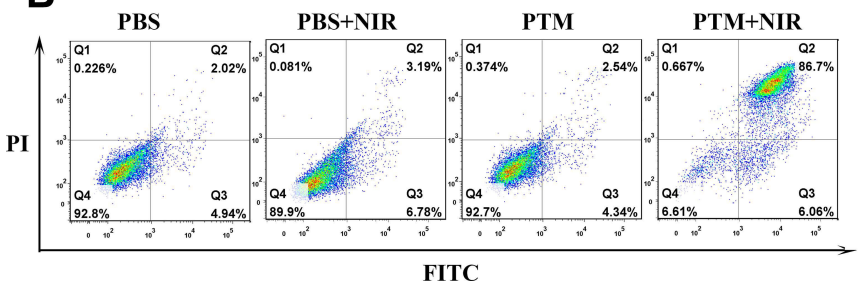

After the UV-vis absorption and photothermal exploration of PTM NPs, density functional theory (DFT) calculation was performed (Figure 1G). The highest occupied molecular orbital (HOMO) of the phenothiazine group were occupied by electron clouds, whereas the 2-dicyanomethylenethiazole unit dominated the lowest unoccupied molecular orbital (LUMO) with the narrow band gap (Eg $=1.51 \mathrm{eV})$.

\section{Laser-Induced Thermal Ablation of PTM NPs in vitro}

It is fundamental for phototherapy agents to exhibit low dark toxicity but high toxicity under laser radiation. Mouse breast cancer $4 \mathrm{~T} 1$ cell line donated by the Department of Cell Biology, School of Basic Medical Sciences, Southern Medical University that were purchased from the National Infrastructure of Cell Line Resource (China) were employed in this study. ACCK-8 analysis for toxicity evaluation of PTM NPs in 4T1 cells without irradiation demonstrated that these agents had excellent biocompatibility (Figure S11). On the other hand, the cell viability decreased to $27 \%$ when incubation of PTM NPs $(200 \mu \mathrm{g} /$ $\mathrm{mL}$ ) upon $808 \mathrm{~nm}$ irradiating $\left(1.5 \mathrm{~W} / \mathrm{cm}^{2}, 10 \mathrm{~min}\right)$, suggesting PTM NPs may be amenable to photoactive therapy.

Fluorescent images of 4T1 cells dyed with calcein AM and propidium iodide after PTM NPs with photothermal therapy treatment are illustrated in Figure 2A, strong red

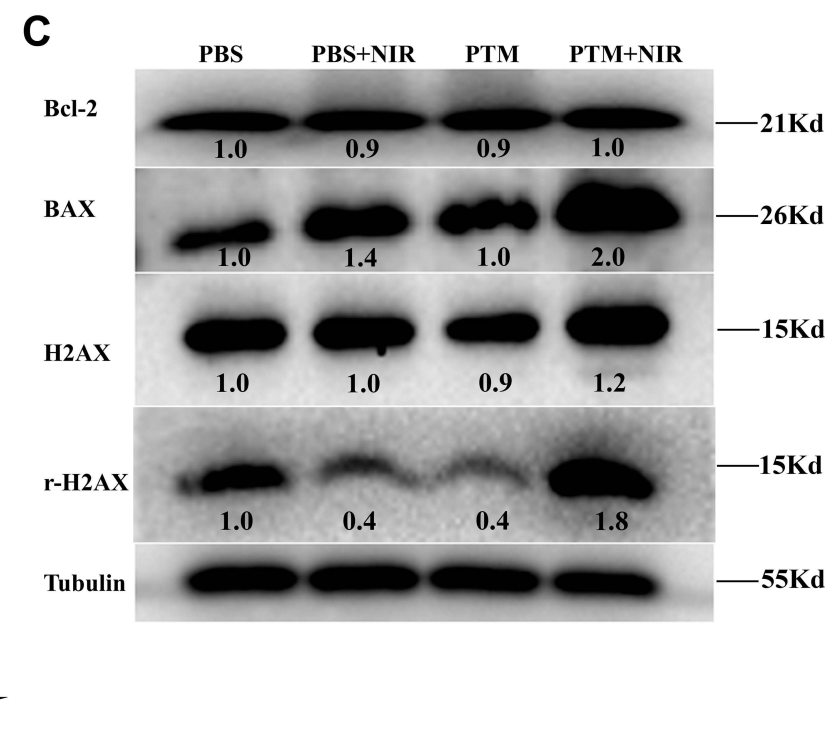

Figure 2 (A) AM/PI fluorescence imaging of 4TI cells with PTM NPs $(200 \mu \mathrm{g} / \mathrm{mL})$ in the absence or presence of NIR laser irradiation $\left(808 \mathrm{~nm}\right.$, I.5 W/cm ${ }^{2}$, I0 min). The scale is $100 \mu \mathrm{m}$. (B) Representative FCM profiles of 4 TI cells with PTM NPs $(80 \mu \mathrm{g} / \mathrm{mL})$ in the absence or presence of NIR laser irradiation $(808 \mathrm{~nm}$, I.5 W/cm², $5 \mathrm{~min})$. (C) PTT induced the $\mathrm{Bcl}-2, \mathrm{BAX}, \mathrm{H} 2 \mathrm{AX}$, and $\gamma \mathrm{H} 2 \mathrm{AX}$ expression in $4 \mathrm{TI}$ cells after different treatments. 
fluorescence in cells was observed. However, the group of PTM NPs treated or laser alone has almost no red fluorescence (Figure S12), confirming that the cells were killed after co-incubation with PTM NPs and $808 \mathrm{~nm}$ laser irradiation. Apoptosis assay was then employed to investigate the apoptosis and necrosis rate induced by activated PTT with PTM NPs. As displayed in Figures 2B, 4T1 cells treated with PTM NPs upon $808 \mathrm{~nm}$ laser irradiation displayed an apoptotic ratio to be $\sim 92.76 \%$, which is notably superior to that in other groups.

To clarify the cell death pathway in charge of the observed effects, we surveyed the effects of PTM NPs on Bax, Bcl-2, H2AX, and $\gamma \mathrm{H} 2 \mathrm{AX}$. It is well known that $\mathrm{Bcl}-2$ has anti-apoptotic properties, which is usually opposite to Bax. The balance between Bcl-2 and Bax is crucial for cell apoptosis or survival. ${ }^{61-63} \gamma \mathrm{H} 2 \mathrm{AX}$ indicates the specific phosphorylation at serine 139 of the histone $\mathrm{H} 2 \mathrm{AX}$, the generation of double-strand breaks always induces $\gamma \mathrm{H} 2 \mathrm{AX}$ formation. ${ }^{64,65}$ Therefore, the expression of $\gamma \mathrm{H} 2 \mathrm{AX}$ reflects the DNA damage. We analyzed their levels by Western blotting (WB) analysis with tubulin as the loading control. As exhibited in Figure 2C, the expression of Bax improved, while the Bcl-2 level diminished and the ratio between Bcl-2 and Bax declined when 4T1 cells were treated with PTM NPs and $808 \mathrm{~nm}$ laser radiation, which proved that apoptosis of cells increased. Moreover, PTM NPs + NIR laser irradiation significantly increased the expression of $\gamma \mathrm{H} 2 \mathrm{AX}$, confirming that this PTT treatment results in DNA damage. These datas verified the hypothesis that DNA damage might be the anticancer mechanism for PTT.

\section{In vivo Photothermal Properties}

Five-week-old female nude mouse $(\mathrm{n}=20)$ were purchased from the Animal Center of Southern Medical University. All animal procedures were performed in accordance with

\section{A}
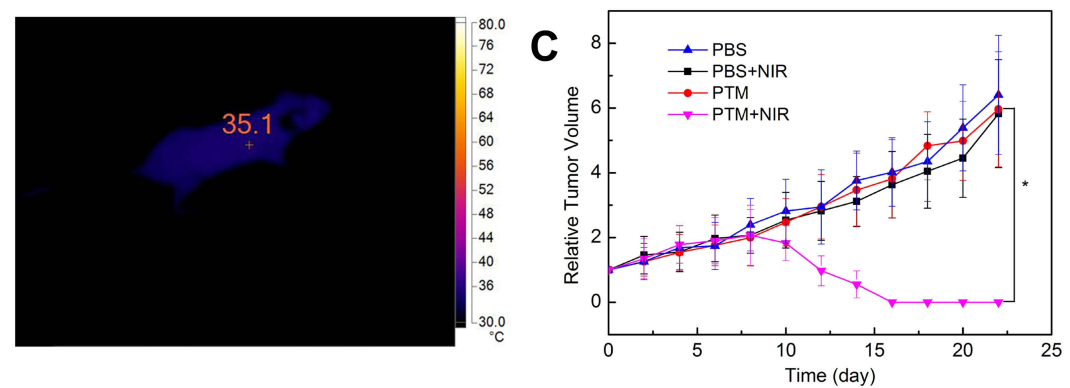

B

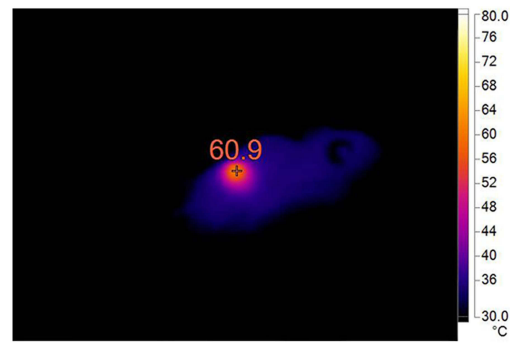

G

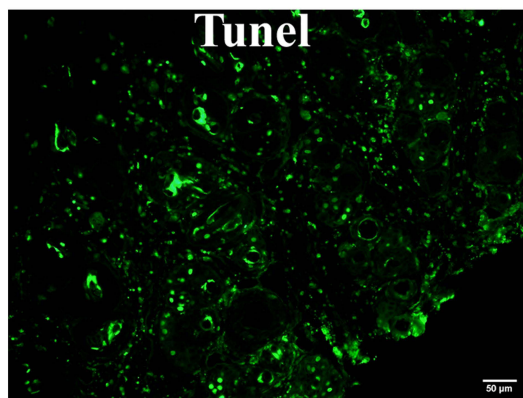

D
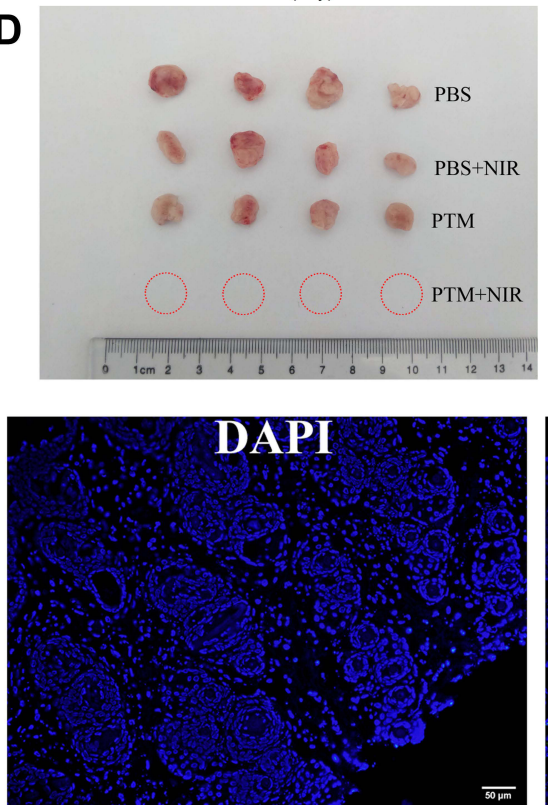
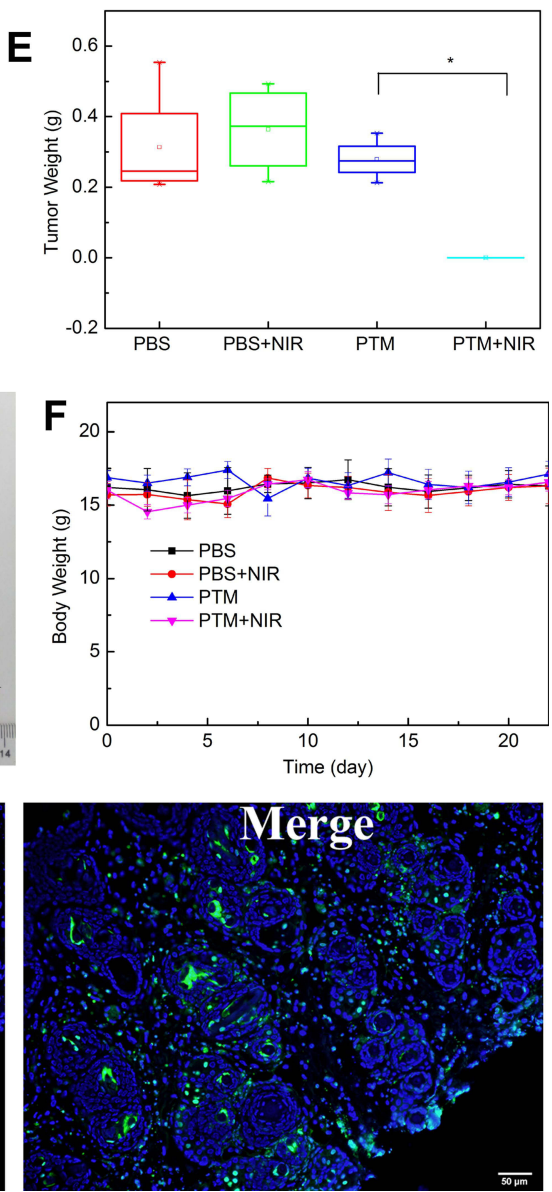

Figure 3 (A) and (B) Tumor temperature of PTM + NIR laser irradiation group before and after irradiation $\left(808 \mathrm{~nm}, 1.0 \mathrm{~W} / \mathrm{cm}^{2}, 1 \mathrm{~min}\right)$. (C) Tumor growth curves of mice after different treatments. Results are expressed as mean \pm S.E. $* P<0.05$ compared with the PTM+NIR laser irradiation group. (D) Photographs of the dissected tumors after different treatment, (E) Dissected tumors weight after different treatment. Results are expressed as mean \pm S.E. $*$ P $<0.05$ compared with the PTM+NIR laser irradiation group. (F) Body weight curve of mice after PTM+NIR laser irradiation group. (G) TUNEL assay of the tumor tissues after PTT. 
the "Guidelines for the Care and Use of Laboratory Animals of Southern Medical University" and were approved by the Animal Ethics Committee of Southern Medical University.

Finally, in vivo photothermal efficacy was conducted by post-intratumoral injection. Firstly, temperature changes in tumor was monitored by infrared thermography during irradiation (Figure $3 \mathrm{~A}$ and $\mathrm{B}$ ). The tumor surface temperature of the $\mathrm{PTM}$ group rose to $60.9^{\circ} \mathrm{C}$ upon 808 $\mathrm{nm}$ laser irradiation for $1 \mathrm{~min}$ which provided adequate hyperthermia to kill cancer cells. What's more, the tumor surface temperature of the PBS injection group was roughly unchanged upon $808 \mathrm{~nm}$ laser irradiation for 1 min (Figure S13).

After that, 4T1 tumor-bearing mice were randomly batched into four groups: PBS control group (group A), $808 \mathrm{~nm}$ laser irradiation only (group B), PTM NPs (2.5 mg/kg) treated only (group C), PTM NPs (2.5 mg/ $\mathrm{kg}$ ) and NIR laser irradiation ( $\left.808 \mathrm{~nm}, 2.0 \mathrm{~W} / \mathrm{cm}^{2}, 5 \mathrm{~min}\right)$ (group D). Changes in tumor size and body weight were monitored in all the groups. Notably, no apparent abnormal body weight changes and other signs of toxic side effects were observed in all treated groups. As shown in
Figure 3C-E, groups A, B, and $\mathrm{C}$ all displayed minimal tumor inhibition, implying that laser exposure or PTM NPs themselves have little influence on the tumor growth. However, Group D (PTM NPs upon $808 \mathrm{~nm}$ laser irradiation) displayed tumor ablation, confirming the apoptosisinduced potential, which is well in consonance with the flow cytometry analysis. All the groups demonstrated similar body weight variation curves (Figure 3F), indicating that PTM NPs exhibited slightly side effects. Additionally, apparent apoptosis and necrosis were observed in PTT treated group (Figure 3G), indicated that PTM NPs plus $808 \mathrm{~nm}$ laser radiation triggers cell apoptosis and necrosis.

\section{Light Induces DNA Damage in Cancer Cells by the PTM NPs}

Owing to the excellent photothermal performance of PTM NPs observed in vitro and vivo experiments, we wonder the action anti-tumor mechanisms of PTM NPs. To further understand the action mechanisms of PTM NPs, RNA-seq analysis was carried out to identify differentially regulated genes after PTM NPs treatment (Figure 4A and B). Enriched in metabolism pathways,
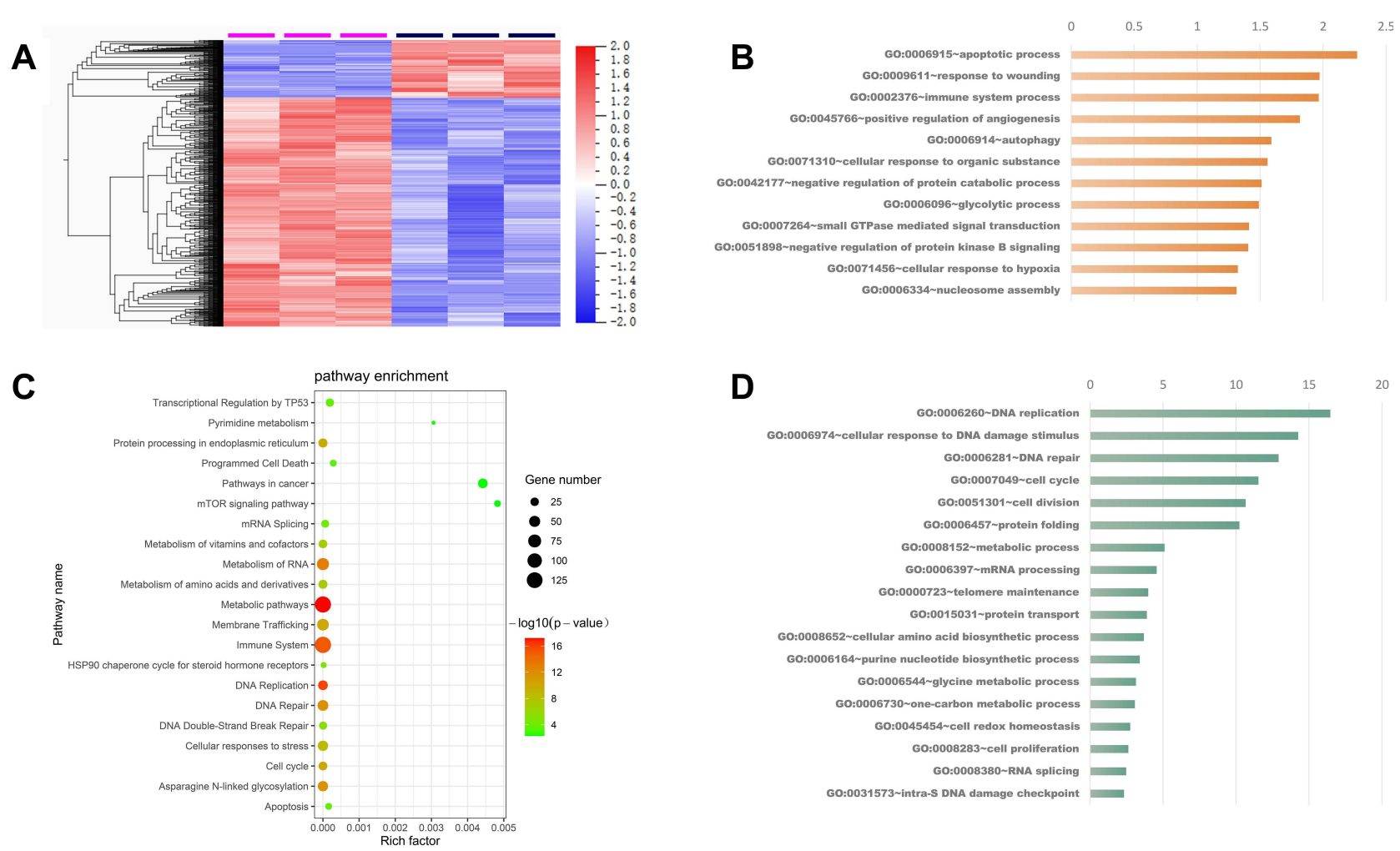

Figure 4 (A) Heatmap showed the differential expressed genes after PTM NPs treated. (B) Enriched KEGG pathway analysis of differentially expressed genes after PTM NPs treated. (C) and (D) Enriched GO biological process of up-regulated genes (orange) and down-regulated genes (blue) in PTM NPs treated group. The X-axis of the histogram is - $\log 10$ (P-value) of individual terms calculated by right-sided hypergeometric test and corrected with Bonferroni. GO categories are indicated on Y-axis. 
immune system, DNA repair, cell cycle, and mRNA splicing was observed (Figure 4C and D). The expression of genes involved in the apoptotic process, glycolytic process, and immune system process were upregulated. In contrast, the genes involved in DNA repair, cell cycle, metabolic process, protein folding, and cellular amino acid biosynthetic process were downregulated. The results confirmed that the enriched expressed genes are involved in the cell apoptosis pathways, including the focal adhesion, adherend junction, and Wnt signaling pathway after PTM NPs + NIR laser irradiation treatment.

\section{The Biocompatibility of PTM NPS}

The in vivo toxicology of PTM NPs was examined. Hematoxylin and eosin (H\&E) staining was carried out to measure the histological changes of main organs (Figure 5A). No obvious pathological variation of major organs was observed in all the groups, confirmed that the excellent biocompatibility of PTM NPs. Moreover, blood biochemical analyses and hematology markers were investigated to examine the in vivo toxicity of PTM NPs (Figure 5B). No apparent distinction was detected in PTM NPs treated groups. All these results highlight the good biological compatibility and safety of PTM NPs.

\section{Conclusion}

In conclusion, a new NIR-absorbing A- $\pi$-D type organic chromophore (PTM) based on 2-dicyanomethylenethiazole was prepared for laser-induced thermal ablation tumors. The encapsulation of PTM into an amphiphilic copolymer F-127 yields PTM NPs with good biocompatibility, excellent photostability, and high photothermal conversion efficiency (56.9\%). Furthermore, the therapeutic mechanism and RNA-seq and biological analysis revealed that hyperthermia induces cell apoptosis via DNA-damage. In vivo investigation, PTM NPs demonstrated excellent tumor ablation under irradiation. All these results confirmed that the A- $\pi-\mathrm{D}$ structured small molecule had great potential for NIR-triggered phototherapy of the tumor.

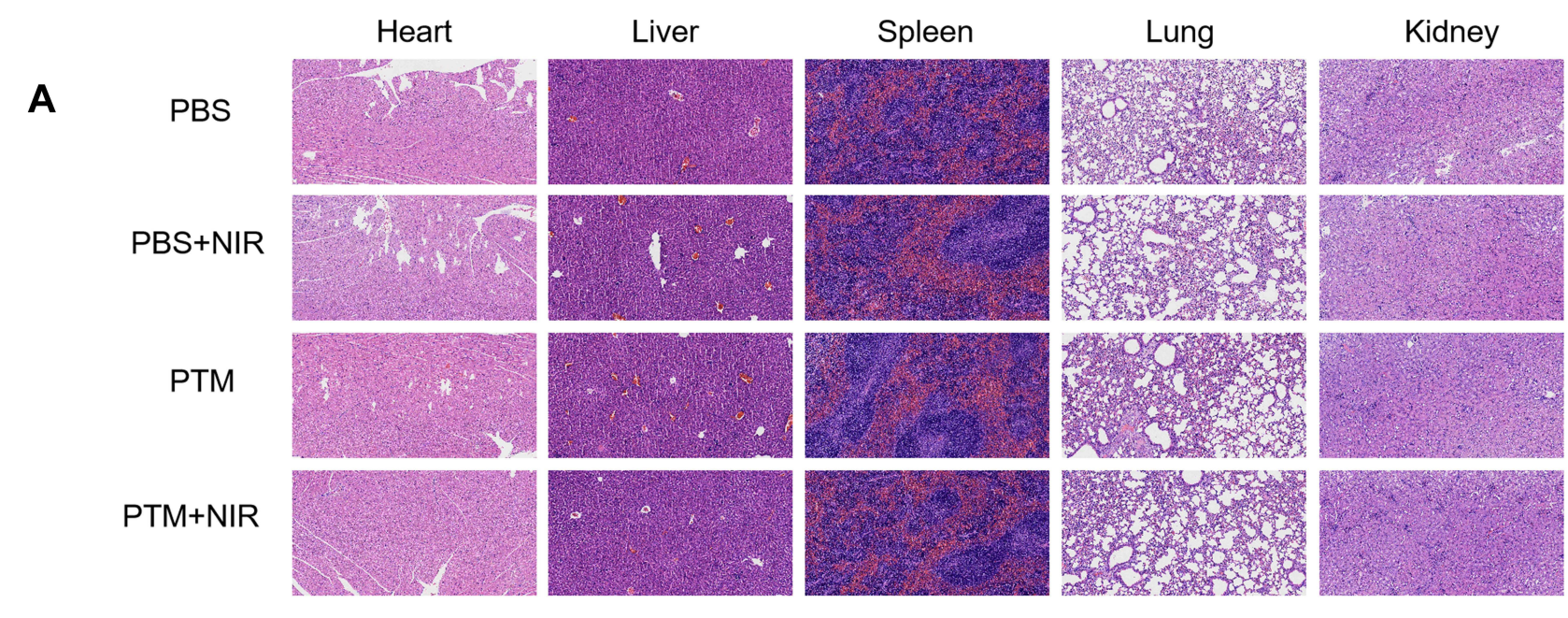

B
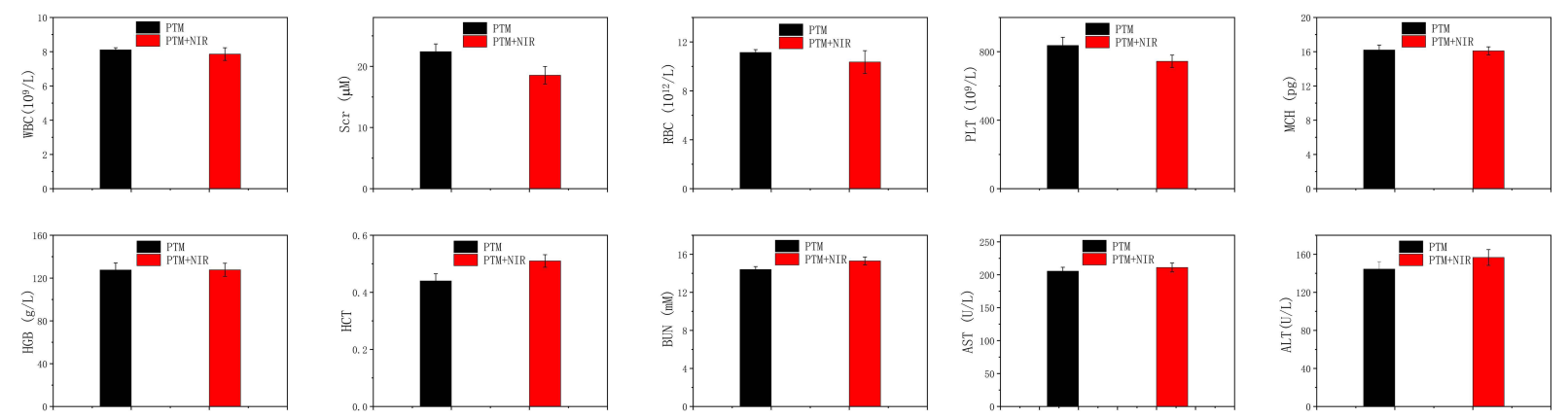

Figure 5 (A) H\&E staining images of major organs in the mice after the intravenous injection. (B) The influence of PTM NPs on the hematopoietic system of mice. 


\section{Acknowledgments}

We gratefully acknowledge the the National Natural Science Foundation of China (81671749 and 81671905) for financial support.

\section{Disclosure}

The authors report no conflicts of interest in this work.

\section{References}

1. Zhao F, Su H, Han X, et al. Tumor Thermal Ablation Enhancement by Micromaterials. Curr Drug Deliv. 2017;14(3):323-333. doi:10.2174/1567201813666160108114208

2. Webb H, Lubner MG, Hinshaw JL. Thermal ablation. Semin Roentgenol. 2011;46(2):133-141. doi:10.1053/j.ro.2010.08.002

3. Izzo F, Granata V, Grassi R, et al. Radiofrequency Ablation and Microwave Ablation in Liver Tumors: an Update. Oncologist 2019;24(10):e990-e1005. doi:10.1634/theoncologist.2018-0337

4. Larghi A, Rimbaș M, Tringali A, et al. Endoscopic radiofrequency biliary ablation treatment: a comprehensive review. Dig Endosc. 2019;231(3):245-255. doi:10.1111/den.13298

5. Vogl TJ, Nour-Eldin NA, Hammerstingl RM, et al. Microwave Ablation (MWA): basics, Technique and Results in Primary and Metastatic Liver Neoplasms. Rofo. 2017;189(11):1055-1066. doi:10.1055/s-0043-117410

6. Hernández JI, Cepeda MF, Valdés F, et al. Microwave ablation: state-of-the-art review. Onco Targets Ther. 2015;8:1627-1632. doi:10.2147/OTT.S81734

7. van den Bijgaart RJ, Eikelenboo DC, Hoogenboom M, et al. Thermal and mechanical high-intensity focused ultrasound: perspectives on tumor ablation, immune effects and combination strategies. Cancer Immunol Immunother. 2017;66(2):247-258. doi:10.1007/s00262-0161891-9

8. Elhelf IAS, Albahar H, Shah U, et al. High intensity focused ultrasound: the fundamentals, clinical applications and research trends. Diagn Inter Imaging. 2018;99(6):349-359. doi:10.1016/j. diii.2018.03.001

9. Jilian RM, Rachel SE, Emily SD. Elucidating the Fundamental Mechanisms of Cell Death Triggered by Photothermal Therapy. ACS Nano. 2015;9(1):6-11. doi:10.1021/acsnano.5b00021

10. Chen Q, Wen J, Li H, et al. Recent advances in different modal imaging-guided photothermal therapy. Biomaterials. 2016;106:144-166.

11. Wang K, Xiang Y, Pan W, et al. Dual-targeted photothermal agents for enhanced cancer therapy. Chem Sci. 2020;11(31):8055-8072. doi:10.1039/D0SC03173A

12. Liu Y, Bhattarai P, Dai Z, et al. Photothermal therapy and photoacoustic imaging via nanotheranostics in fighting cancer. Chem Soc Rev. 2019;48(7):2053-2108.

13. Guo X, Cao B, Wang $C$, et al. In vivo photothermal inhibition of methicillin-resistant Staphylococcus aureus infection by in situ templated formulation of pathogen-targeting phototheranostics. Nanoscale. 2020;12(14):7651-9659. doi:10.1039/D0NR00181C

14. Wang C, Zhao W, Cao B, et al. Biofilm-Responsive Polymeric Nanoparticles with Self-Adaptive Deep Penetration for In Vivo Photothermal Treatment of Implant Infection. Chem Materials. 2020;32(18):7725-7738. doi:10.1021/acs.chemmater.0c02055

15. Wang Z, Zhan M, Li W, et al. Photoacoustic Cavitation-Ignited Reactive Oxygen Species to Amplify Peroxynitrite Burst by Photosensitization-free Polymeric Nanocapsules. Angew Chem Int Ed. 2021;60(9):4720-4731. doi:10.1002/anie.202013301
16. Sztandera K, Gorzkiewicz M, Klajnert-Maculewicz B. Gold Nanoparticles in Cancer Treatment. Mol Pharmaceut. 2019;16 (1):1-23. doi:10.1021/acs.molpharmaceut.8b00810

17. Chung YJ, Kim J, Park CB. Photonic Carbon Dots as an Emerging Nanoagent for Biomedical and Healthcare Applications. ACS Nano. 2020;14(6):6470-6497. doi:10.1021/acsnano.0c02114

18. Liu S, Pan X, Two-Dimensional LH. Nanomaterials for Photothermal Therapy. Angew Chem Int Ed. 2020;59(15):5890-5900. doi:10.1002/ anie. 201911477

19. Sun H, Lv F, Liu L, et al. Conjugated Polymer Materials for Photothermal Therapy. Adv Ther. 2018;1(6):1800057. doi:10.1002/ adtp. 201800057

20. Chen Y, Fan Z, Zhan Z, et al. Two-Dimensional Metal Nanomaterials: synthesis, Properties, and Applications. Chem Rev. 2018;118(13):6409-6455. doi:10.1021/acs.chemrev.7b00727

21. Lin W, Li Y, Zhang W, et al. Near-Infrared Polymeric Nanoparticles with High Content of Cyanine for Bimodal Imaging and Photothermal Therapy. ACS Appl Mater Interfaces. 2016;8 (37):24426-24432. doi:10.1021/acsami.6b07103

22. Su S, Ding Y, Li Y, et al. Integration of photothermal therapy and synergistic chemotherapy by a porphyrin self-assembled micelle confers chemosensitivity in triple-negative breast cancer. Biomaterials. 2016;80:169-178. doi:10.1016/j.biomaterials.2015.11.058

23. Alifu N, Zebibul A, Qi J, et al. Single-Molecular Near-Infrared-II Theranostic Systems: ultrastable Aggregation-Induced Emission Nanoparticles for Long-Term Tracing and Efficient Photothermal Therapy. ACS Nano. 2018;12(11):11282-11293. doi:10.1021/ acsnano.8b05937

24. Ji C, Cheng W, Yuan Q, et al. From Dyestuff Chemistry to Cancer Theranostics: the Rise of Rylenecarboximides. Acc Chem Res. 2019;52(8):2266-2277. doi:10.1021/acs.accounts.9b00221

25. Liu C, Zhang S, Li J, et al. Water-Soluble, NIR-Absorbing Quaterrylenediimide Chromophore for Photoacoustic Imaging and Efficient Photothermal Cancer Therapy. Angew Chem Int Ed. 2019;58(6):1638-1642. doi:10.1002/anie.201810541

26. Zhang S, Guo W, Wei J, et al. Terrylenediimide-Based Intrinsic Theranostic Nanomedicines with High Photothermal Conversion Efficiency for Photoacoustic Imaging-Guided Cancer Therapy. ACS Nano. 2017;11(4):3797-3805. doi:10.1021/acsnano.6b08720

27. Wang H, Chan J, Shi M, et al. A Dual-Targeted Organic Photothermal Agent for Enhanced Photothermal Therapy. Angew Chem Int Ed. 2019;58(4):1057-1061. doi:10.1002/anie.201811273

28. Zhang R, Xu Y, Zhang Y, et al. Rational design of a multifunctional molecular dye for dual-modal NIR-II/photoacoustic imaging and photothermal therapy. Chem Sci. 2019;10(36):8348-8353. doi:10.1039/C9SC03504D

29. Zhou B, Li Y, Niu G, et al. Near-Infrared Organic Dye-Based Nanoagent for the Photothermal Therapy of Cancer. ACS App Mater Interfaces. 2016;8(44):29899-29905. doi:10.1021/ acsami.6b07838

30. Zeng X, Xiao Y, Lin J, et al. Near-Infrared II Dye-Protein Complex for Biomedical Imaging and Imaging-Guided Photothermal Therapy. $A d v$ Healthcare Mater. 2018;7(18):1800589. doi:10.1002/adhm.201800589

31. Wang D, Lee MMS, Xu WH, et al. Boosting Non-Radiative Decay to Do Useful Work: development of a Multi-Modality Theranostic System from an AIE gen. Angew Chem Int Ed. 2019;58 (17):5628-5632. doi:10.1002/anie.201900366

32. Chen J, Wen K, Chen H, et al. Achieving High-Performance Photothermal and Photodynamic Effects upon Combining D-A Structure and Nonplanar Conformation. Small. 2020;16 (17):2000909. doi:10.1002/smll.202000909

33. Cai Y, Liang P, Tang Q, et al. Diketopyrrolopyrrole-Triphenylamine Organic Nanoparticles as Multifunctional Reagents for Photoacoustic Imaging-Guided Photodynamic/Photothermal Synergistic Tumor Therapy. ACS Nano. 2017;211(1):1054-1063. doi:10.1021/ acsnano.6b07927 
34. Wang Y, Huang X, Tang Y, et al. A light-induced nitric oxide controllable release nano-platform based on diketopyrrolopyrrole derivatives for $\mathrm{pH}$-responsive photodynamic/ photothermal synergistic cancer therapy. Chem Sci. 2018;9(42):8103. doi:10.1039/ C8SC03386B

35. Guo B, Huang Z, Shi Q, et al. Organic Small Molecule Based Photothermal Agents with Molecular Rotors for Malignant Breast Cancer Therapy. Adv Funct Mater. 2020;30(5):1907093. doi:10.1002/adfm.201907093

36. Duan Y, Hu D, Guo B, et al. Nanostructural Control Enables Optimized Photoacoustic-Fluorescence-Magnetic Resonance Multimodal Imaging and Photothermal Therapy of Brain Tumor. Adv Funct Mater. 2019;30(1):1907077. doi:10.1002/adfm.201907077

37. Liu S, Zhou X, Zhang H, et al. Molecular Motion in Aggregates: manipulating TICT for Boosting Photothermal Theranostics. $J$ Am Chem Soc. 2019;141(13):5359-5368. doi:10.1021/jacs.8b13889

38. Alifu N, Zebibula A, Qi J, et al. Single-Molecular Near-Infrared-II Theranostic Systems: ultrastable Aggregation-Induced Emission Nanoparticles for Long-Term Tracing and Efficient Photothermal Therapy. ACS Nano. 2018;12(11):11282-11293.

39. He Z, Zhao L, Zhang Q, et al. An Acceptor-Donor-Acceptor Structured Small Molecule for Effective NIR Triggered Dual Phototherapy of Cancer. Adv Funct Mater. 2020;30(16):1910301. doi:10.1002/adfm.201910301

40. Ni JS, Zhang X, Yang G, et al. Photoinduced Nonadiabatic Decay-guided Molecular Motor Triggers Effective Photothermal Conversion for Hyperthermia Cancer Therapy. Angew Chem Int Ed. 2020;59(28):11298-11302. doi:10.1002/anie.202002516

41. Andreu R, Galán E, Orduna J, et al. Aromatic/Proaromatic Donors in 2-Dicyanomethylenethiazole Merocyanines: from Neutral to Strongly Zwitterionic Nonlinear Optical Chromophores. Chem Eur J. 2011;17 (3):826-838. doi:10.1002/chem.201002158

42. Heichert C, Hartmann H. Synthesis and characterization of long wavelength absorbing donor/acceptor-substituted methine dyes. Z Naturforsch. 2016;71(6):651-658. doi:10.1515/znb-2016-0009

43. Chen Z, Xia Q, Zhou Y, et al. 2-Dicyanomethylenethiazole based NIR absorbing organic nanoparticles for photothermal therapy and photoacoustic imaging. J Mater Chem B. 2019;7(25):3950-3957. doi:10.1039/C9TB00808J

44. Bürckstümmer H, Tulyakova EV, Deppisch M, et al. Efficient Solution-Processed Bulk Heterojunction Solar Cells by Antiparallel Supramolecular Arrangement of Dipolar Donor-Acceptor Dyes. Angew Chem Int Ed. 2011;50(49):11628-11632. doi:10.1002/ anie. 201105133

45. Esteban SG, de la Cruz P, Aljarilla A, et al. Panchromatic Push-Pull Chromophores based on Triphenylamine as Donors for Molecular Solar Cells. Org Lett. 2013;13(19):5362-5365. doi:10.1021/ ol202242j

46. Chen W, Wang X, Zhao B, et al. CuS-MnS 2 nano-flowers for magnetic resonance imaging guided photothermal/photodynamic therapy of ovarian cancer through necroptosis. Nanoscale. 2019;11 (27):12983-12989. doi:10.1039/C9NR03114F

47. Yuan $\mathrm{M}, \mathrm{Xu} \mathrm{S}$, Zhang Q, et al. Biocompatible porous $\mathrm{Co}_{3} \mathrm{O}_{4}$ nanoplates with intrinsic tumor metastasis inhibition for multimodal imaging and DNA damage-mediated tumor synergetic photothermal/ photodynamic therapy. Chem Eng J. 2020;394:124874. doi:10.1016/ j.cej.2020.124874

48. Murray D, Milas L, Meyn RE. DNA damage produced by combined hyperglycemia and hyperthermia in two mouse fibrosarcoma tumors in vivo. Int $J$ Radiat Oncol Biol Phys. 1984;10(9):1679-1682. doi:10.1016/0360-3016(84)90527-3

49. Anai H, Maehara Y, Sugimachi K. In situ nick translation method reveals DNA strand scission in HeLa cells following heat treatment. Cancer Lett. 1988;40(1):33-38. doi:10.1016/0304-3835(88)90259-5
50. Muenyi CS, States VA, Masters JH, Fan TW, Helm CW, States JC. Sodium arsenite and hyperthermia modulate cisplatin-DNA damage responses and enhance platinum accumulation in murine metastatic ovarian cancer xenograft after hyperthermic intraperitoneal chemotherapy (HIPEC). J Ovarian Res. 2011;4(1):9. doi:10.1186/17572215-4-9

51. Mantso T, Goussetis G, Franco R, Botaitis S, Pappa A, Panayiotidis M. Effects of hyperthermia as a mitigation strategy in DNA damage-based cancer therapies. Semin Cancer Biol. 2016;3738:96-105. doi:10.1016/j.semcancer.2016.03.004

52. Shao J, Ji S, Li X, et al. Thiophene-Inserted Aryl-Dicyanovinyl Compounds: the Second Generation of Fluorescent Molecular Rotors with Significantly Redshifted Emission and Large Stokes Shift. Eur J Org Chem. 2011;2011(30):6100-6109. doi:10.1002/ ejoc. 201100891

53. Men X, Wang F, Chen H, et al. Ultrasmall Semiconducting Polymer Dots with Rapid Clearance for Second Near-Infrared Photoacoustic Imaging and Photothermal Cancer Therapy. Adv Funct Mater. 2020;30(24):1909673. doi:10.1002/adfm.201909673

54. Wu F, Chen L, Yue L, et al. Small-Molecule Porphyrin-Based Organic Nanoparticles with Remarkable Photothermal Conversion Efficiency for in Vivo Photoacoustic Imaging and Photothermal Therapy. ACS Appl Mater Interfaces. 2019;11(24):21408-21416. doi:10.1021/acsami.9b06866

55. Wu F, Yue L, Cheng K, et al. Facile Preparation of Phthalocyanine-Based Nanodots for Photoacoustic Imaging and Photothermal Cancer Therapy In Vivo. ACS Biomater Sci Eng. 2020;6(9):5230-5239. doi:10.1021/acsbiomaterials.0c00684

56. Li S, Sun Z, Deng G, et al. Dual-modal imaging-guided highly efficient photothermal therapy using heptamethine cyanine-conjugated hyaluronic acid micelles. Biomater Sci. 2017;5 (6):1122-1129. doi:10.1039/C7BM00230K

57. Chen M, Fang X, Tang S, et al. Polypyrrole nanoparticles for high-performance in vivo near-infrared photothermal cancer therapy. Chem Commun. 2012;48(71):8934-8936. doi:10.1039/ c2cc34463g

58. Liu Y, Ai K, Liu J, et al. Dopamine-melanin colloidal nanospheres: an efficient near-infrared photothermal therapeutic agent for in vivo cancer therapy. Adv Mater. 2013;25(9):1353-1359. doi:10.1002/ adma.201204683

59. Yang T, Liu L, Deng Y, et al. Ultrastable Near-Infrared Conjugated-Polymer Nanoparticles for Dually Photoactive Tumor Inhibition. Adv Mater. 2017;29(31):1700487. doi:10.1002/ adma.201700487

60. Yin W, Yan L, Yu J, et al. High-throughput synthesis of single-layer $\mathrm{MoS}_{2}$ nanosheets as a near-infrared photothermal-triggered drug delivery for effective cancer therapy. ACS Nano. 2014;8 (7):6922-6933. doi:10.1021/nn501647j

61. Tacar O, Sriamornsak P, Dass CR. Doxorubicin: an update on anticancer molecular action, toxicity and novel drug delivery systems. $J$ Pharm Pharmacol. 2013;65(2):157-170. doi:10.1111/j.20427158.2012.01567.x

62. Reed JC. Bcl-2 and the regulation of programmed cell death. $J$ Cell Biol. 1994;124(1-2):1-6. doi:10.1083/jcb.124.1.1

63. Oltvai ZN. Bcl-2 heterodimerizes in vivo with a conserved homolog, Bax, that accelerates programmed cell death. Cell. 1993;74 (4):609-619. doi:10.1016/0092-8674(93)90509-O

64. Rogakou EP, Pilch DR, Orr AH, et al. Double-stranded breaks induce histone H2AX phosphorylation on Serine 139. J Biol Chem. 1998;273(10):5858-5868. doi:10.1074/jbc.273.10.5858

65. Anastasios G, Constantinos V, George C, et al. Genome Instability and $\gamma$ H2AX. Int $J$ Mol Sci. 2017;18(9):1979. doi:10.3390/ ijms18091979 


\section{Publish your work in this journal}

The International Journal of Nanomedicine is an international, peerreviewed journal focusing on the application of nanotechnology in diagnostics, therapeutics, and drug delivery systems throughout the biomedical field. This journal is indexed on PubMed Central, MedLine, CAS, SciSearch ${ }^{\mathbb{R}}$, Current Contents ${ }^{\mathbb{B}} /$ Clinical Medicine,
Journal Citation Reports/Science Edition, EMBase, Scopus and the Elsevier Bibliographic databases. The manuscript management system is completely online and includes a very quick and fair peer-review system, which is all easy to use. Visit http://www.dovepress.com/ testimonials.php to read real quotes from published authors.

Submit your manuscript here: https://www.dovepress.com/international-journal-of-nanomedicine-journal 